\title{
Surgical Timing and Outcomes of Unilateral Versus Bilateral Superior Cavopulmonary Anastomosis: An Analysis of Pediatric Heart Network Public Databases
}

\author{
Jeffrey M. Shuler ${ }^{1}$ (1) $\cdot$ Chris Statile $e^{2,3} \cdot$ Haleh Heydarian ${ }^{2,3} \cdot$ David G. Lehenbauer $^{2,3,4} \cdot$ Garick D. Hill $^{2,3}$
}

Received: 28 July 2020 / Accepted: 18 December 2020 / Published online: 8 January 2021

○ The Author(s), under exclusive licence to Springer Science+Business Media, LLC part of Springer Nature 2021

\begin{abstract}
Requiring bilateral superior cavopulmonary anastomosis (bSCPA) instead of unilateral superior cavopulmonary anastomosis (uSCPA) could influence surgical timing and outcomes. We compared surgical timing and outcomes for patients who underwent uSCPA to those who underwent bSCPA through use of the Pediatric Heart Network's public datasets for the Infant Single Ventricle trial and Single Ventricle Reconstruction trial. There was no statistically significant difference in median age at SCPA (158 vs. 150 days, $p=0.68$ ), hospital length of stay (LOS) ( 7 vs. 7 days, $p=0.74$ ), intensive care unit (ICU) LOS ( 4 vs. 5 days, $\mathrm{p}=0.53$ ), time requiring ventilator support ( 2 vs. 2 days, $\mathrm{p}=0.51$ ), or oxygen saturation at discharge ( 82 vs. $81 \%, p=0.22$ ) between the uSCPA and bSCPA groups, respectively. However, sub-analysis comparing only those who underwent early SCPA, at $<120$ days of age, revealed significantly longer hospital LOS ( 8 vs. 13 days, $p=0.04$ ), ICU LOS ( 5 vs. 11 days, $p=0.01$ ), and time requiring ventilator support ( 2 vs. 4 days, $p=0.03$ ) for the early bSCPA group when compared to the early uSCPA group. A multivariable logistic regression revealed bSCPA to be the only significant predictor of prolonged hospital LOS for patients who underwent early SCPA (odds ratio 4.1, 95\% CI 1.2-14.2). Overall, there was no difference in surgical timing or outcome measures between uSCPA and bSCPA. However, early bSCPA, performed at $<120$ days, had worse outcome measures than early uSCPA. Delaying elective bSCPA until at least 120 days of age could minimize morbidity in infants with bilateral superior venae cavae.
\end{abstract}

Keywords Superior cavopulmonary anastomosis $\cdot$ Glenn $\cdot$ Single ventricle

\section{Introduction}

The superior cavopulmonary anastomosis (SCPA) operation, also known as the bidirectional Glenn, involves connecting the superior vena cava (SVC) directly to the pulmonary arteries (PAs) and is typically used as part of a staged palliation in patients with a functionally univentricular heart.

Jeffrey M. Shuler

jeffrey.shuler@cchmc.org

1 Department of Pediatrics, Cincinnati Children's Hospital, 3333 Burnet Ave, Cincinnati, OH 45229, USA

2 Cincinnati Children's Hospital, Heart Institute, Cincinnati, $\mathrm{OH}, \mathrm{USA}$

3 Department of Pediatrics, University of Cincinnati College of Medicine, Cincinnati, OH, USA

4 Department of Surgery, University of Cincinnati College of Medicine, Cincinnati, OH, USA
It is usually performed around four to 6 months of age as a second-stage palliative procedure toward the ultimate goal of Fontan completion, also known as total cavopulmonary anastomosis [1,2]. For patients with a single SVC, the procedure is a unilateral superior cavopulmonary anastomosis (USCPA) and for patients with bilateral SVCs, the procedure is a bilateral superior cavopulmonary anastomosis (bSCPA). In a bSCPA, each superior vena cava must be anastomosed to its ipsilateral PA, thus making bSCPA a more complex procedure.

Multiple studies have compared uSCPA and bSCPA. Some have shown bSCPA to be a risk factor for thrombus formation and unfavorable alterations in PA growth [3], while others have directly contradicted this and shown no such risk increase [4]. Some have shown bSCPA to be safe with subsequent Fontan success [4, 5], while others have shown that bSCPA patients have higher rates of post-operative complications, Fontan failure, and early mortality when compared to uSCPA patients [6]. There are conflicting data 
on whether bSCPA patients have worse outcomes than uSCPA patients, although many factors and hypotheses have been proposed [3, 4, 6, 7]. Age at surgery and anatomic complexity have been shown to be associated with adverse outcomes [7, 8]. SCPA timing and perioperative practices vary by center [9-11]. Although optimal timing of SCPA has been evaluated in some populations, it has not been directly compared between uSCPA and bSCPA [12].

The Infant Single Ventricle (ISV) and Single Ventricle Reconstruction (SVR) trials, conducted by the Pediatric Heart Network, generated extensive databases of patients with single ventricle lesions who underwent surgical palliation. The ISV trial examined effects of angiotensinconverting enzyme inhibition in these infants and the SVR trial compared survival of newborns randomized to either a modified Blalock-Taussig shunt or a right ventricle to pulmonary artery shunt as part of the Norwood procedure [13, 14]. Through the use of databases from these studies, we sought to compare timing of surgery for patients who have undergone uSCPA versus bSCPA and to assess for any correlation between surgical timing and outcomes.

\section{Material and Methods}

Data from the publicly available and de-identified datasets of the ISV and SVR trials were utilized. The ISV trial was a randomized double-blind placebo-controlled trial, conducted at 10 centers in the United States and Canada from 2003 to 2007 to evaluate the efficacy of enalapril in patients with single ventricle anatomy. The database includes data for 185 infants from $<45$ days old through 14 months [13]. The SVR trial was a randomized trial, conducted at 15 centers from 2005 to 2009, with a database that includes data for 549 infants with single ventricle anatomy who underwent surgical palliation randomized to two different shunt types at the time of the Norwood operation. Patients were enrolled as newborns prior to Norwood palliation and followed to 14 months of age [14]. Common variables from both datasets were merged to form a combined dataset. Patients who underwent SCPA were included, while those with interrupted IVC requiring Kawashima $(n=11)$ were excluded. The combined dataset created for the full cohort of patients included 481 uSCPA patients and 70 bSCPA patients. A subanalysis was performed for an early SCPA cohort, defined as SCPA performed at less than 120 days of age. This age cutoff was chosen as it represents approximately the first quartile for SCPA timing [7]. This early SCPA cohort included 81 early uSCPA patients and 15 early bSCPA patients. Demographics and outcome variables for patients who underwent uSCPA were compared to those of patients who underwent bSCPA.
Table 1 Demographic characteristics, full cohort

\begin{tabular}{llll}
\hline & uSCPA $(\mathrm{n}=481)$ & $\mathrm{bSCPA}(\mathrm{n}=70)$ & P value \\
\hline $\begin{array}{l}\text { Birth weight, g } \\
\text { Gestational age, }\end{array}$ & $3200(2844-3518)$ & $3229(2880-3640)$ & 0.74 \\
$\quad$ weeks & & $39(37-40)$ & 0.83 \\
Male, n (\%) & $316(65.7)$ & $47(67.1)$ & 0.89 \\
White race, n (\%) & $391(82)$ & $52(74.3)$ & 0.41 \\
$\begin{array}{l}\text { Hispanic ethnicity, } \\
\text { n (\%) }\end{array}$ & $19(16.7)$ & $12(17.1)$ & 0.87 \\
Age at SCPA, days & $158(128-187)$ & $150(128-197)$ & 0.68 \\
\hline
\end{tabular}

USCPA unilateral superior cavopulmonary anastomosis, $b S C P A$ bilateral superior cavopulmonary anastomosis

Table 2 Demographic characteristics, SCPA at $<120$ days

\begin{tabular}{llll}
\hline & uSCPA $(\mathrm{n}=81)$ & bSCPA $(\mathrm{n}=15)$ & P value \\
\hline $\begin{array}{l}\text { Birth weight, g } \\
\text { Gestational age, }\end{array}$ & $3255(2925-3600)$ & $3180(2935-3325)$ & 0.42 \\
$\quad$ weeks & & $38(38-39)$ & 0.98 \\
Male, n (\%) & $64(79)$ & $13(86.7)$ & 0.73 \\
White race, n (\%) & $66(81.5)$ & $13(86.7)$ & 0.53 \\
$\begin{array}{l}\text { Hispanic ethnicity, } \\
\quad 66(82.5)\end{array}$ & $12(80)$ & 0.73 \\
$\quad$ Age at SCPA, days & $103(92-108)$ & $93(78-110)$ & 0.11 \\
\hline
\end{tabular}

Statistical analyses were performed using StataIC15 (Stata Corp, College Station TX). Continuous variables are expressed as median with interquartile range. Categorical data are expressed as frequency with percentage. Fisher's exact test was performed for categorical data and the Mann-Whitney U test was performed for continuous data. A multivariable logistic regression was performed for early SCPA patients to determine factors associated with hospital LOS greater than 10 days.

\section{Results}

For the full cohort $(\mathrm{n}=551)$ there were 188 (34\%) females with $443(80 \%)$ white, 81 (15\%) black/African American and $91(17 \%)$ of Hispanic ethnicity. The median gestational age was 38 (38-39) weeks and median birth weight was 3.2 $(2.8-3.5) \mathrm{kg}$. There were 70 patients $(12.7 \%)$ who underwent bSCPA. Comparison of demographic data for the full cohort (Table 1) and sub-analysis for the early SCPA cohort (Table 2) revealed no significant differences between the uSCPA and bSCPA groups in median birth weight, median gestational age, sex, race, ethnicity, or age at time of SCPA. Among the uSCPA patients, 454 (94.4\%) had undergone a Norwood operation prior to SCPA compared to $60(85.7 \%)$ of the bSCPA patients $(\mathrm{p}=0.007)$. 
Table 3 Post-operative outcome variables, full cohort

\begin{tabular}{llll}
\hline & uSCPA $(\mathrm{n}=481)$ & bSCPA $(\mathrm{n}=70)$ & P value \\
\hline Time on ventilator, days & $2(1-3)$ & $2(1-4)$ & 0.51 \\
ICU LOS, days & $4(3-7)$ & $5(3-7)$ & 0.53 \\
Hospital LOS, days & $7(5-11)$ & $7(5-11)$ & 0.74 \\
SpO2 at discharge, $\%$ & $82(79-85)$ & $81(77-84)$ & 0.22 \\
Mortality by 14 months, $\mathrm{n}(\%)$ & $33(6.9)$ & $4(5.7)$ & 0.98 \\
\hline
\end{tabular}

$I C U$ intensive care unit, $\mathrm{LOS}$ length of stay, $\mathrm{SpO} 2$ peripheral oxygen saturation
Table 4 Post-operative outcome variables, SCPA at $<120$ days

\begin{tabular}{llll}
\hline & uSCPA $(\mathrm{n}=81)$ & bSCPA $(\mathrm{n}=15)$ & P value \\
\hline Time on ventilator, days & $2(1-4)$ & $4(2-11)$ & 0.03 \\
ICU LOS, days & $5(4-8)$ & $11(5-23)$ & 0.01 \\
Hospital LOS, days & $8(7-18)$ & $13(10-31)$ & 0.04 \\
SpO2 at discharge, \% & $81(79-83)$ & $80(75-82)$ & 0.16 \\
$\begin{array}{l}\text { Mortality by 14 months, } \\
\text { n (\%) }\end{array}$ & $8(9.9)$ & $2(13.3)$ & 0.65 \\
\hline
\end{tabular}

Analysis of post-operative outcome variables for the full cohort revealed no significant difference between the uSCPA and bSCPA groups with regards to days requiring ventilator support, days in the intensive care unit (ICU), post-operative hospital length of stay (LOS), oxygen saturation (SpO2) at discharge, or mortality up to 14 months (Table 3). Sub-analysis of the same outcome variables for the early SCPA cohort revealed that patients who underwent early bSCPA spent significantly more days on the ventilator, more days in the ICU, and more days in the hospital post-operatively than those who underwent early uSCPA, but there was no difference in $\mathrm{SpO} 2$ at discharge or mortality up to 14 months (Table 4). When comparing post-operative hospital LOS in only those patients who had undergone a Norwood operation prior to their SCPA, there was no difference between the uSCPA and bSCPA patients $(13.7 \pm 21.4$ days vs. $14.4 \pm 35.8$ days, $\mathrm{p}=0.7)$.
Scatterplots of age at SCPA and post-operative length of stay are shown for the uSCPA (Fig. 1) and bSCPA groups (Fig. 2). Multivariable logistic regression for early SCPA patients who had prolonged hospital LOS revealed that, among those undergoing an early SCPA, those who underwent bSCPA had an odds ratio of 4.1 for prolonged hospital LOS compared to those who underwent uSCPA. Birth weight, gestational age, sex, race and ethnicity were not significantly associated with prolonged LOS (Table 5).

Use of cardiopulmonary bypass and time was only collected for the SVR trial. In the 390 eligible patients of all ages in this dataset, bypass was used in 384 with a median cardiopulmonary bypass time of 85 (64-118) minutes for the 344 who underwent uSCPA and 102 (71-157) minutes for the 44 who underwent bSCPA $(p=0.06)$.

\section{Discussion}

This study demonstrated that timing of stage II single ventricle palliation and short-term post-operative outcomes did not differ significantly between uSCPA and bSCPA in patients from the ISV and SVR trials. The lack of difference in short-term outcomes between patients undergoing bSCPA versus uSCPA is similar to the findings of Kogon et al. [15]. However, they did find a significant difference in age and weight between these groups, with the bSCPA patients being almost 30 days older and $0.6 \mathrm{~kg}$ larger in their single institution study. A prior evaluation of the ISV cohort for factors
Fig. 1 Hospital LOS by age at uSCPA. Scatter plot displaying relationship between age at uSCPA and post-uSCPA hospital length of stay. Black dots represent patients who underwent uSCPA at $<120$ days of age and gray dots represent patients who underwent uSCPA at $>120$ days of age

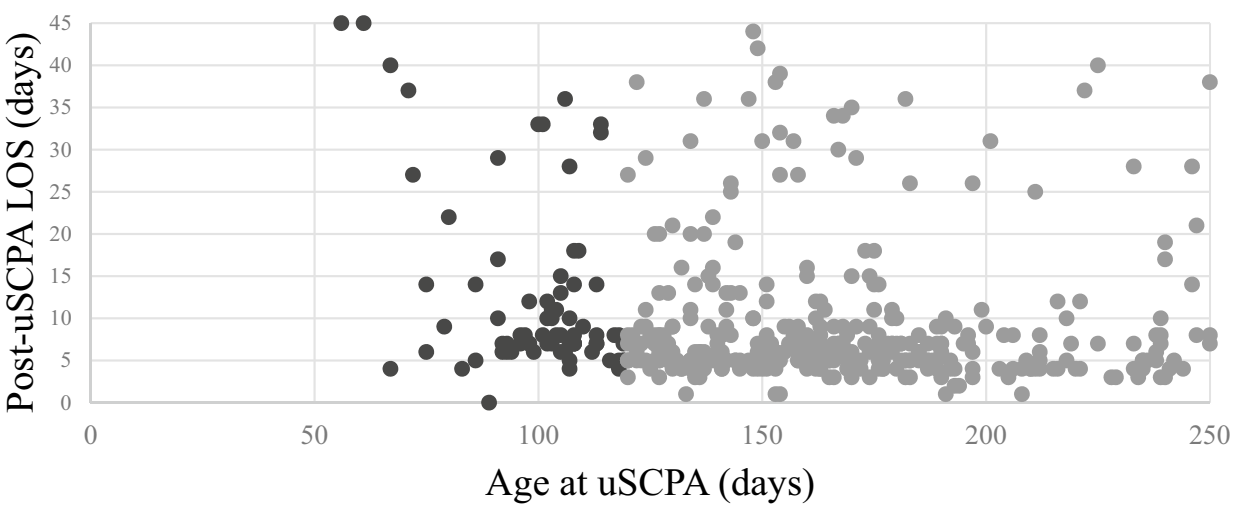

Age at uSCPA (days) 
Fig. 2 Hospitals LOS by age at bSCPA. Scatter plot displaying relationship between age at bSCPA and post-bSCPA hospital LOS. Black dots represent patients who underwent bSCPA at $<120$ days of age and gray dots represent patients who underwent bSCPA at $>120$ days of age

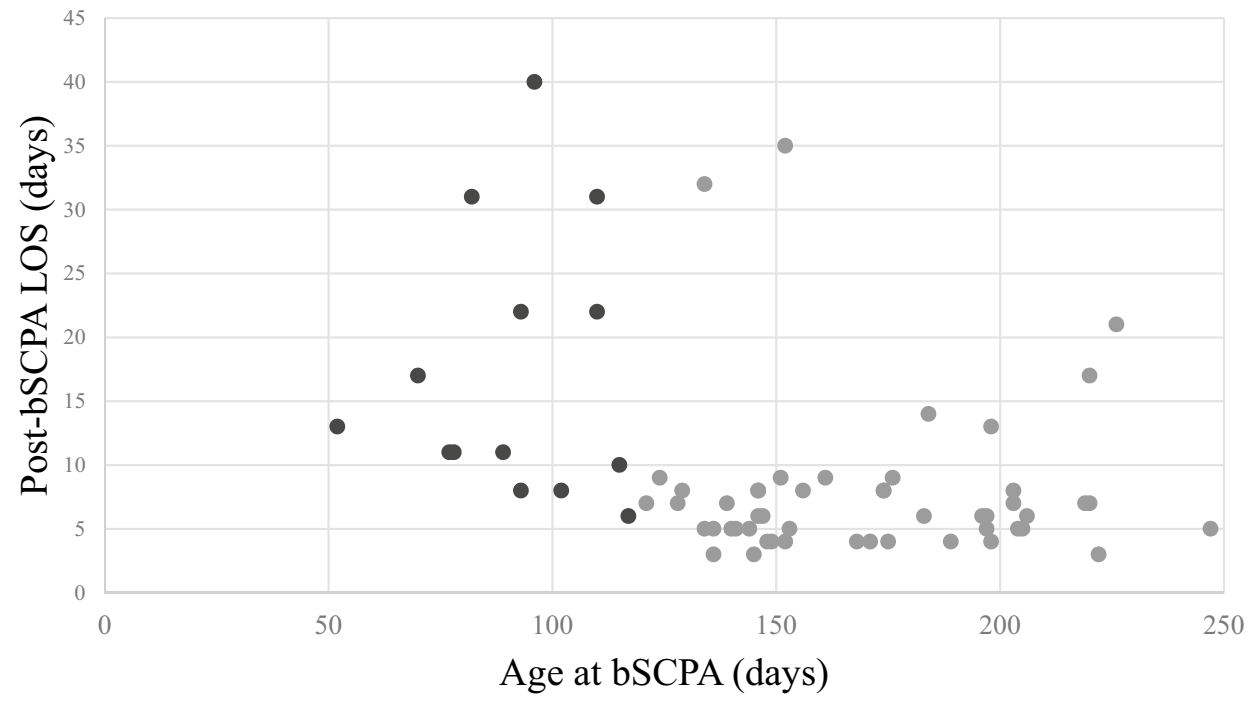

Table 5 Multivariable logistic regression, early SCPA with prolonged hospital LOS

\begin{tabular}{lll}
\hline & Odds ratio $(95 \%$ CI $)$ & P value \\
\hline Sex & $0.45(0.13-1.6)$ & 0.2 \\
Race & $0.91(0.59-1.4)$ & 0.7 \\
Ethnicity & $0.49(0.15-1.6)$ & 0.2 \\
Birth weight & $1(0.998-1.001)$ & 0.8 \\
Gestational age & $1.1(0.7-1.8)$ & 0.6 \\
SCPA type & $4.1(1.2-14.2)$ & 0.03 \\
\hline
\end{tabular}

that influenced timing of SCPA did not specifically evaluate bSCPA versus uSCPA but found that younger age at SCPA was associated with increased pre-operative complexity and pre-SCPA morbidity [7]. Meza et al. determined that 2-year survival after the Norwood procedure was greatest when SCPA was performed at $>3$ months but optimal age differed by risk group [12]. In evaluating longer term outcomes, after discharge from SCPA, we did not find any difference in mortality between uSCPA and bSCPA out to 14 months of age. Iyer at el. similarly found no significant difference in longer term mortality between these groups in a cohort undergoing their SCPA between 1982 and 1998 [3].

Our study is not the first to show an association between early SCPA and prolonged ICU stay, hospitalization, and duration of mechanical ventilation [16, 17]. Others have also identified potential disadvantages of an early SCPA [18]. However, these concerns must be balanced against the risk of prolonging the period of shunt-dependent physiology and increased vulnerability to sudden cardiac death that exists while awaiting second-stage palliation [19]. Centers with a later median age at SCPA following Stage 1 Norwood have indeed been shown to have higher rates of interstage mortality [20]. Furthermore, the age at which SCPA is considered early remains a moving target. Even extremely early SCPA, at less than 8 weeks, has been shown to have good outcomes in some patients with worsening cyanosis and heart failure, challenging the notion that young age alone should contraindicate SCPA [21]. Part of this change may be improved growth for these patients making surgery technically feasible at a younger age $[22,23]$.

Our study is unique in that it represents multi-institution data comparing timing and outcomes of SCPA in populations randomized for other studies. This study also identified poorer short-term outcomes after early bSCPA, defined as $<120$ days of age, compared to early uSCPA. The reasons for this finding could have multiple contributing factors. While it was not statistically significant in the SVR cohort alone, the median bypass time for bSCPA was almost 20 min longer than for uSCPA, regardless of surgical timing. This may have a more significant effect on post-operative recovery in the younger and smaller cohort, as both have been associated with worse outcomes after SCPA [7, 24]. This is compounded by the slightly younger age at SCPA in the bSCPA versus the uSCPA group in our study, although again, this did not reach statistical significance. It is possible that some institutions seek to delay bSCPA, as seen in the study by Kogon et al., and therefore may reserve early bSCPA for only those with unmanageable physiology, such as severe desaturation or inability to gain weight. In contrast, these institutions may feel more comfortable with early uSCPA for those who are doing well. However, some difference in age between the groups in the full cohort would be expected if this were the case. Furthermore, the fact that uSCPA patients were more likely to have undergone a Norwood than were bSCPA patients, rather than vice versa, suggests that bSCPA was not merely a surrogate for greater complexity or increased pre-operative risk. 


\section{Limitations}

Our study's comparisons were limited to the demographics, diagnostic information, and outcomes that were measured in the ISV and SVR trials. By combining the two datasets, measured variables were primarily limited even further to those that were shared between both trials. While speculations can be made, we do not have data that definitively elucidate what specific factors led to certain patients undergoing surgery earlier than others. Furthermore, while these data sets combined did produce a robust data set, even so, the bSCPA group was relatively small in size with only 70 total bSCPA patients and 15 early bSCPA patients. Finally, while there were mortality data out to 14 months of age, longer term outcomes were not available for comparison.

\section{Conclusions}

Timing of SCPA and hospital outcomes are similar between patients with unilateral and bilateral superior venae cavae. However, early bSCPA, performed before 120 days of life, is less well tolerated than early uSCPA with increased duration of mechanical ventilation and hospitalization. Delaying elective SCPA until at least 120 days of age could minimize short-term morbidity in infants with bilateral superior venae cavae, but additional delay compared to patients with a single superior vena cava is not necessary.

Acknowledgements The NIH/NHLBI Pediatric Heart Network Infant Single Ventricle trial and Single Ventricle Reconstruction Trial datasets were used in preparation of this work. Data were downloaded from https://www.pediatricheartnetwork.org//pud_login.asp?study_id=ISV and from https://www.pediatricheartnetwork.com/pud_login.asp?study _id=SVR on 5/20/2019.

Funding The study was not funded by any agency or through any specific grants.

Data Availability Not applicable.

Code Availability Not applicable.

\section{Compliance with Ethical Standards}

Conflict of interest Authors have no conflicts of interest to declare.

Ethical Approval The study was approved by the institutional review board and ethics committee.

Consent to Participate Not applicable.

Consent for Publication Not applicable.

\section{References}

1. Ohye RG, Schranz D, D'Udekem Y (2016) Current therapy for hypoplastic left heart syndrome and related single ventricle lesions. Circulation 134(17):1265-1279

2. Rao PS (2013) Consensus on timing of intervention for common congenital heart diseases: Part II - cyanotic heart defects. Indian J Pediatr 80(8):663-674

3. Iyer GK, Van Arsdell GS, Dicke FP et al (2000) Are bilateral superior vena cavae a risk factor for single ventricle palliation? Ann Thorac Surg 70(3):711-716

4. Ando Y, Fukae K, Hirayama K, Oe M, Iwai T (2014) Impact of bilateral superior venae cavae on outcome of staged Fontan procedure. Ann Thorac Surg 98(6):2187-2193

5. El Midany AAH, Mostafa EA, Mansour SA et al (2017) Bilateral bidirectional Glenn: outcome of off-pump technique. Interact Cardiovasc Thorac Surg 25(5):745-749

6. Keizman E, Tejman-Yarden S, Mishali D et al (2019) The bilateral bidirectional glenn operation as a risk factor prior to fontan completion in complex congenital heart disease patients. World J Pediatr Congenit Heart Surg 10(2):174-181

7. Cnota JF, Allen KR, Colan S et al (2013) Superior cavopulmonary anastomosis timing and outcomes in infants with single ventricle. J Thorac Cardiovasc Surg 145(5):1288-1296

8. Silvilairat S, Pongprot Y, Sittiwangkul R et al (2008) Factors influencing survival in patients after bidirectional Glenn shunt. Asian Cardiovasc Thorac Ann 16(5):381-386

9. Herrmann JL, Brown JW (2019) The superior cavopulmonary connection: history and current perspectives. World J Pediatr Congenit Heart Surg 10(2):216-222

10. Agarwal A, Firdouse M, Brar N et al (2018) Incidence and management of thrombotic and thromboembolic complications following the superior cavopulmonary anastomosis procedure: a literature review. Clin Appl Thromb Hemost 24(3):405-415

11. Eckhauser A, Pasquali SK, Ravishankar C et al (2018) Variation in care for infants undergoing the Stage II palliation for hypoplastic left heart syndrome. Cardiol Young 28(10):1109-1115

12. Meza JM, Hickey EJ, Blackstone EH et al (2017) The optimal timing of stage 2 palliation for hypoplastic left heart syndrome: an analysis of the pediatric heart network single ventricle reconstruction trial public data set. Circulation 136(18):1737-1748

13. Hsu DT, Zak V, Mahony L et al (2010) Enalapril in infants with single ventricle: results of a multicenter randomized trial. Circulation 122(4):333-340

14. Ohye RG, Sleeper LA, Mahony L et al (2010) Comparison of shunt types in the Norwood procedure for single-ventricle lesions. N Engl J Med 362(21):1980-1992

15. Kogon BE, Plattner C, Leong T et al (2008) The bidirectional Glenn operation: a risk factor analysis for morbidity and mortality. J Thorac Cardiovasc Surg 136(5):1237-1242

16. Jaquiss RD, Ghanayem NS, Hoffman GM et al (2004) Early cavopulmonary anastomosis in very young infants after the Norwood procedure: impact on oxygenation, resource utilization, and mortality. J Thorac Cardiovasc Surg 127(4):982-989

17. Petrucci O, Khoury PR, Manning PB, Eghtesady P (2010) Outcomes of the bidirectional Glenn procedure in patients less than 3 months of age. J Thorac Cardiovasc Surg 139(3):562-568

18. Reddy VM, Liddicoat JR, Hanley FL (1995) Primary bidirectional superior cavopulmonary shunt in infants between 1 and 4 months of age. Ann Thorac Surg 59(5):1120-1125

19. Ghanayem NS, Tweddell JS, Hoffman GM et al (2006) Optimal timing of the second stage of palliation for hypoplastic left heart syndrome facilitated through home monitoring, and the results of early cavopulmonary anastomosis. Cardiol Young 16(Suppl 1):61-66 
20. Hill GD, Rudd NA, Ghanayem NS et al (2016) Center variability in timing of stage 2 palliation and association with interstage mortality: a report from the national pediatric cardiology quality improvement collaborative. Pediatr Cardiol 37(8):1516-1524

21. Slavik Z, Lamb RK, Webber SA et al (1996) Bidirectional superior cavopulmonary anastomosis: how young is too young? Heart 75(1):78-82

22. Hehir DA, Rudd N, Slicker J et al (2012) Normal interstage growth after the norwood operation associated with interstage home monitoring. Pediatr Cardiol 33(8):1315-1322

23. Petit CJ, Fraser CD, Mattamal R et al (2011) The impact of a dedicated single-ventricle home-monitoring program on interstage somatic growth, interstage attrition, and 1-year survival. J Thorac Cardiovasc Surg 142(6):1358-1366

24. Anderson JB, Beekman RH 3rd, Border WL et al (2009) Lower weight-for-age $\mathrm{z}$ score adversely affects hospital length of stay after the bidirectional Glenn procedure in 100 infants with a single ventricle. J Thorac Cardiovasc Surg 138(2):397-404

Publisher's Note Springer Nature remains neutral with regard to jurisdictional claims in published maps and institutional affiliations. 